\title{
The Aquatic Macrophyte Flora of the Pandeiros River Wildlife Sanctuary, Minas Gerais, Brazil
}

\author{
Marco Otávio Dias Pivari ${ }^{1 *}$, Pedro Lage Viana ${ }^{1}$ and Felipe Sá Fortes Leite ${ }^{2}$ \\ 1 Universidade Federal de Minas Gerais, Instituto de Ciências Biológicas, Laboratório de Sistemática Vegetal. Avenida Antônio Carlos 6621. CEP \\ 31270-010. Belo Horizonte, MG, Brazil. \\ 2 Universidade Federal de Minas Gerais, Departamento de Zoologia. Avenida Antônio Carlos 6621. CEP 31270-010. Belo Horizonte, MG, Brazil. \\ * Corresponding author. E-mail: marcopivari@yahoo.com.br
}

\begin{abstract}
The São Francisco River forms one of the main Brazilian hydrographic basins of ca. 645,000 km2. The Pandeiros River is a tributary situated on the left margin of the São Francisco and is considered a strategic component for conservation of biodiversity of that hydrographic basin. An inventory of the aquatic macrophyte flora of the Pandeiros River Wildlife Sanctuary was carried out, using collections of botanical samples and examination of specimens at the BHCB Herbarium. Aquatic environments in the study area were classified as follows: the Pandeiros riverbed, floodplains, oxbow lakes, and swamps. A total of 101 species was inventoried, distributed in 37 families (1 charophytes, 1 liverworts, 3 ferns and 32 angiosperms) and 71 genera. The species were classified into seven life forms, with the amphibian and rafted plants the more representative. The area shows a high diversity in its aquatic macrophytes and has an important role in the conservation of biodiversity of the region.
\end{abstract}

\section{INTRODUCTION}

Aquatic environments correspond to approximately $11 \%$ of the continental area of tropical regions (Rebouças et al. 1999). Brazil, with its largest hydrographic network in the world, has a high degree of habitat diversification, representing ecosystems that make up its natural landscape (Bove et al. 2003). However, several aquatic environments have been drained or eliminated in the last decades as a result of the expansion of agricultural activities, industrial development, urbanization, establishment of hydroelectric power reservoirs, and by attempts to eliminate breeding sites of disease vectors (Esteves 1988). These factors have been responsible for the disappearance of numerous habitats and, therefore, the plant diversity associated with them, potentially influencing fundamental ecologic processes, such as the nutrient cycling and primary productivity of water bodies.

The São Francisco forms one of the main Brazilian hydrographic basins with ca. $645,000 \mathrm{~km}^{2}(7,5 \%$ of the country's area), of great social, economic, cultural, and biological relevance (Kohler 2003). Severe anthropic pressure of the last decades, such as the elimination of riparian forests and the transposition of its waters (a project carried out by the National Integration Ministry of the Brazilian Government) have had a negative impact on its biota that can be readily observed at several of its stretches.

The São Francisco rises in Minas Gerais, where several of its medium to large tributaries are found, accounting for more than $70 \%$ of its water (Kohler 2003). Some of those are located in the northern part of the state, an ecotonal area between the two important Brazilian biogeographic domains, the Caatinga and the Cerrado. A mosaic of different phyto-physiognomies is found in the region (Lombardi et al. 2005), composed of savannas, deciduous forests (associated with limestone outcrops), riparian forests, and several aquatic macrophyte environments, associated with watercourses (Barbosa and Maillard 2010).

Even though the need for special attention to preserve the biodiversity in northern Minas Gerais is strongly felt, very few efforts focusing on documenting its floristic diversity have been made so far. The present study aims to contribute to the knowledge of its regional flora, and more specifically, to the study of aquatic macrophytes of the Pandeiros River Wildlife Sanctuary.

\section{Materials AND Methods}

Study site

The Pandeiros River is a tributary situated on the left margin of the São Francisco and is considered a strategic component in the conservation of biodiversity of that hydrographic basin, particularly with respect to its ichthyofauna (Sato and Godinho 2003). The Pandeiros encompasses the most expressive swamp (known locally as pântano) in the state, extending to up to 5,000 ha during the rainy season (Nunes et al. 2009) and highly favorable to the establishment of aquatic macrophytes. This unique ecosystem is regarded as a priority area for the biodiversity conservation of the Cerrado domain (Conservação Internacional 1999) in the state (Drummond et al. 2005) and has two conservation units: the Pandeiros River Wildlife Sanctuary (with full protection) and the Environmental Protection Area of the Panderios River (with sustainable use).

The Pandeiros River Wildlife Sanctuary is located in the municipality of Januária, Minas Gerais, and is delimited by the coordinates of $15^{\circ} 30^{\prime}$ to $15^{\circ} 42^{\prime} \mathrm{S}$ and $44^{\circ} 35^{\prime}$ to $44^{\circ} 46^{\prime} \mathrm{W}$, occupying a total area of 6,102.75 ha (Figure 1). In accordance with Koppen's (1931) classification, the climate of the region is classified as Aw, moist tropical climate with a dry season during the winter. Temperatures 
range from $16^{\circ} \mathrm{C}$ to $34^{\circ} \mathrm{C}$ (with the annual mean around $24^{\circ} \mathrm{C}$ ), while precipitation varies from 850 to $950 \mathrm{~mm}$, with occasional long dry periods (Ratter et al. 1978). The aquatic environments in the study area can be classified as: the Pandeiros riverbed (PAN), floodplains (FLP), oxbow lakes (OXL), and swamps (SWP). Table 1 summarizes some features of these environments.

\section{Data collection}

The inventory of aquatic macrophytes of the Pandeiros River Wildlife Sanctuary was carried out during the beginning of the rainy season (September 2007) and the dry season (June 2008) in the area. Transportation for the collection of samples in the environments cited in Table 1 was done by motorboat. Fertile material was prepared in accordance with the usual techniques for collecting aquatic macrophytes (Haynes 1984; Ceska et al. 1986) and the specimens were deposited at the BHCB herbarium (acronym follows Thiers 2012). Additional specimens from the study area previously deposited at the BHCB were also included in the compilation.

For species identification, pertinent literature was studied, including taxonomic treatments and guides, as well as comparison with the BHCB herbarium specimens previously determined by experts. Additionally, duplicates were sent to experts for confirmation and/or identification of some species. The classification of the angiosperm families follows APG III (2009); for ferns, Kramer and Green's (1990) classification is adopted; and for charophytes and liverworts, Raven et al. (1990) is followed. Nomenclatural terminology and author abbreviations are

TABLE 1. Aquatic environments of the Pandeiros River Wildlife Sanctuary and their principal features.

\begin{tabular}{llll}
\hline SITES & ACRONYM & FLOODABLE & WATERFLOW \\
\hline Pandeiros riverbed & PAN & Perennial & Lotic \\
Floodplains & FLP & Seasonal & Intermediate \\
Oxbow lakes & OXL & Perennial & Lentic \\
Swamps & SWP & Perennial & Intermediate \\
\hline
\end{tabular}

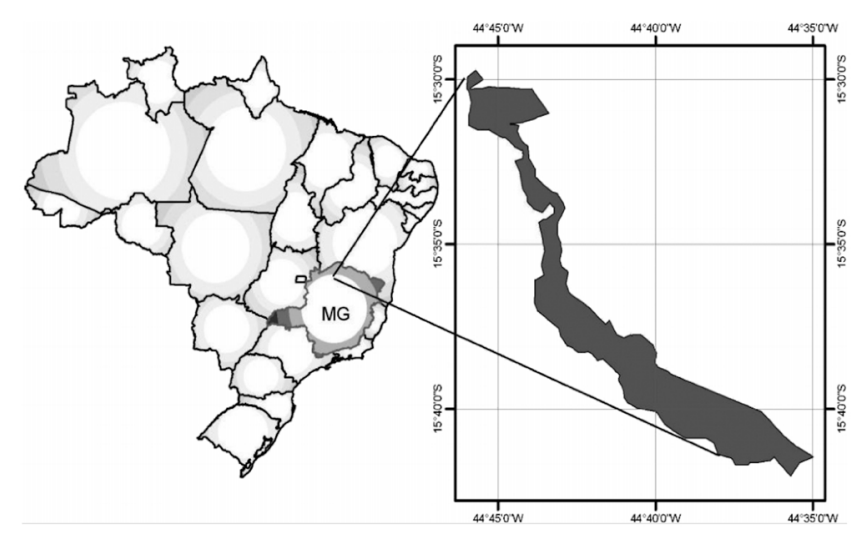

FIgURE 1. Location of the Pandeiros River Wildlife Sanctuary in Minas Gerais (MG), Brazil (extracted from Barbosa and Maillard 2010). according to Brummit and Powell (1992). The life forms names were adopted in accordance with the classification proposed by Irgang et al. (1984), except for plants growing on organic soil (histosoil) of floating meadows, which are classified as rafted plants, as suggested by Pivari et al. (2011). Data for the type of habitat for each species were obtained from exsiccate labels.

\section{RESULTS AND DiscuSSION}

A total of 101 aquatic macrophyte species was inventoried (Figures 2-6). The species are distributed in 37 families: 1 charophytes ( 2 spp.), 1 liverworts ( 1 spp.), 3 ferns (7 spp.) and 32 angiosperms (91 spp.), and 71 genera. The most represented families were Cyperaceae (10 spp.), Poaceae (9 spp.), Asteraceae (7 spp.), Fabaceae, Plantaginaceae and Pontederiaceae (with $6 \mathrm{spp}$. each), Alismataceae, Onagraceae and Rubiaceae (with 5 spp. each). The more speciose genera were Bacopa, Cyperus and Ludwigia (with 5 spp., each), followed by Borreria, Echinodorus and Polygonum (with 3 spp. each).

The inventoried species were classified into seven life forms (Table 2), with the prevalence of the amphibians (42 spp.) and rafted plants (27 spp.). The amphibian species were mostly associated with floodplains and borders of oxbow lakes, where the soil is transitional from dry to flooded during the annual hydrological cycle. The species classified as rafted plants were recorded exclusively in oxbow lakes, environments that gather adequate conditions for the establishment of floating meadows (Duzer 2004) as a result of succession of aquatic plants (Pivari et al. 2008) that accumulate large amounts of floating mulch and culminating in the histosoil formation. Such substrate accounts for the occurrence of several aquatic macrophyte species (Pivari et al. 2008; Pivari et al. 2011). Other life forms sampled in the study were characterized as emerging (12 spp.), fixed submerged (7 spp.), free floating (6 spp.), fixed floating (5 spp.) and free submerged (2 spp., each), as in other Brazilian wetlands (Pott and Pott 2000; Pivari et al. 2011).

In the swamps of the Pandeiros River Wildlife Sanctuary were recorded 14 species distributed into all life forms (except rafted plants, since no floating meadows are found in that environment). Some species, such as Azolla filiculoides, Ceratophyllum demersum, Egeria najas, Ludwigia inclinata, and Nymphaea rudgeana, were observed exclusively in the swamps. Different life forms provide for higher heterogeneity of niches in the establishment and maintenance of aquatic fauna by creating refuges for spawning, nesting, and other specific conditions. The floodplains, on the other hand, accounted for $42 \%$ of the inventoried aquatic macrophyte species. Parts of these areas are significantly affected by cattle grazing, a common farming activity in the region. Therefore, some typical species of disturbed areas were also sampled, such as Eclipta prostrata, Fuirena umbellata and Ludwigia octovalvis (Lorenzi 2000). 


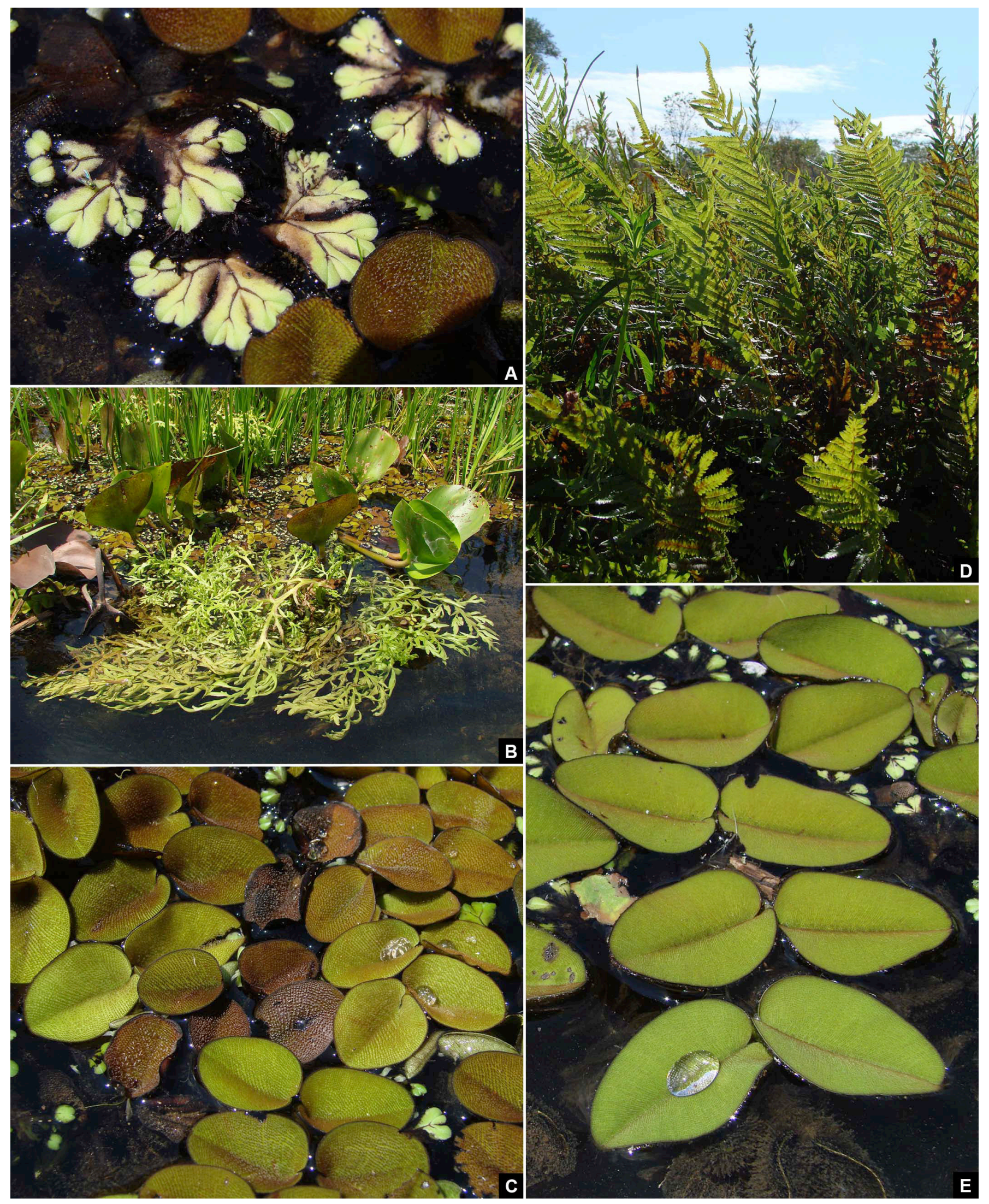

FiguRE 2. Aquatic macrophyte species photographed at the Pandeiros River Wildlife Sanctuary: Liverworts and ferns. A. Ricciocarpus natans (Ricciaceae), B. Ceratopteris pteridoides (Pteridaceae), C. Salvinia auriculata (Salviniaceae), D. Thelypteris interrupta (Thelypteridaceae), E. Salvinia oblongifolia (Salviniaceae). 


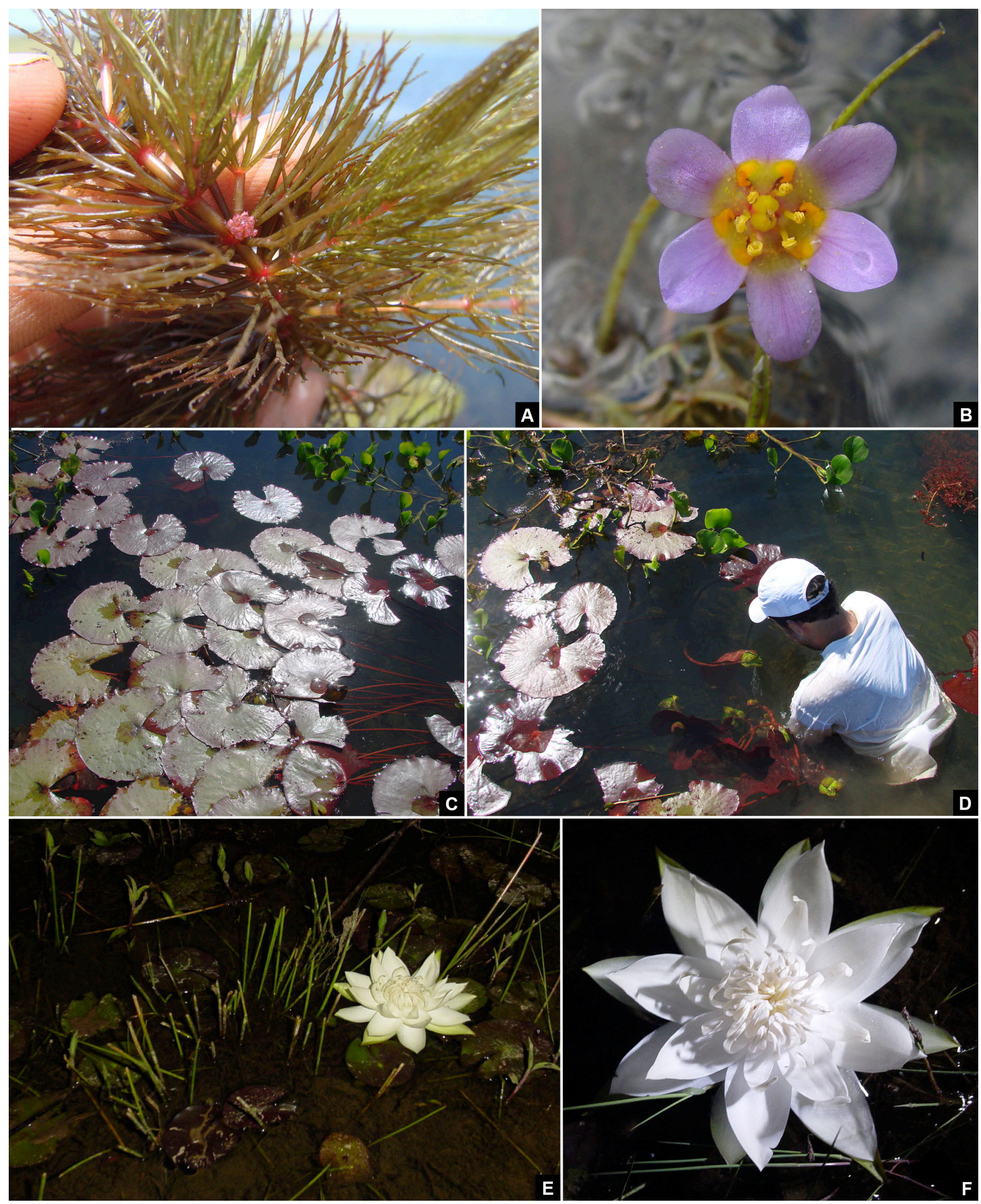

FigURE 3. Aquatic macrophyte species photographed at the Pandeiros River Wildlife Sanctuary: Nymphaeales e Ceratophyllales. A. Ceratophyllum demersum (Ceratophyllaceae), B. Cabomba furcata (Cabombaceae), C-D. Nymphaea rudgeana (Nymphaeaceae), E-F. Nymphaea lingulata (Nymphaeaceae). 

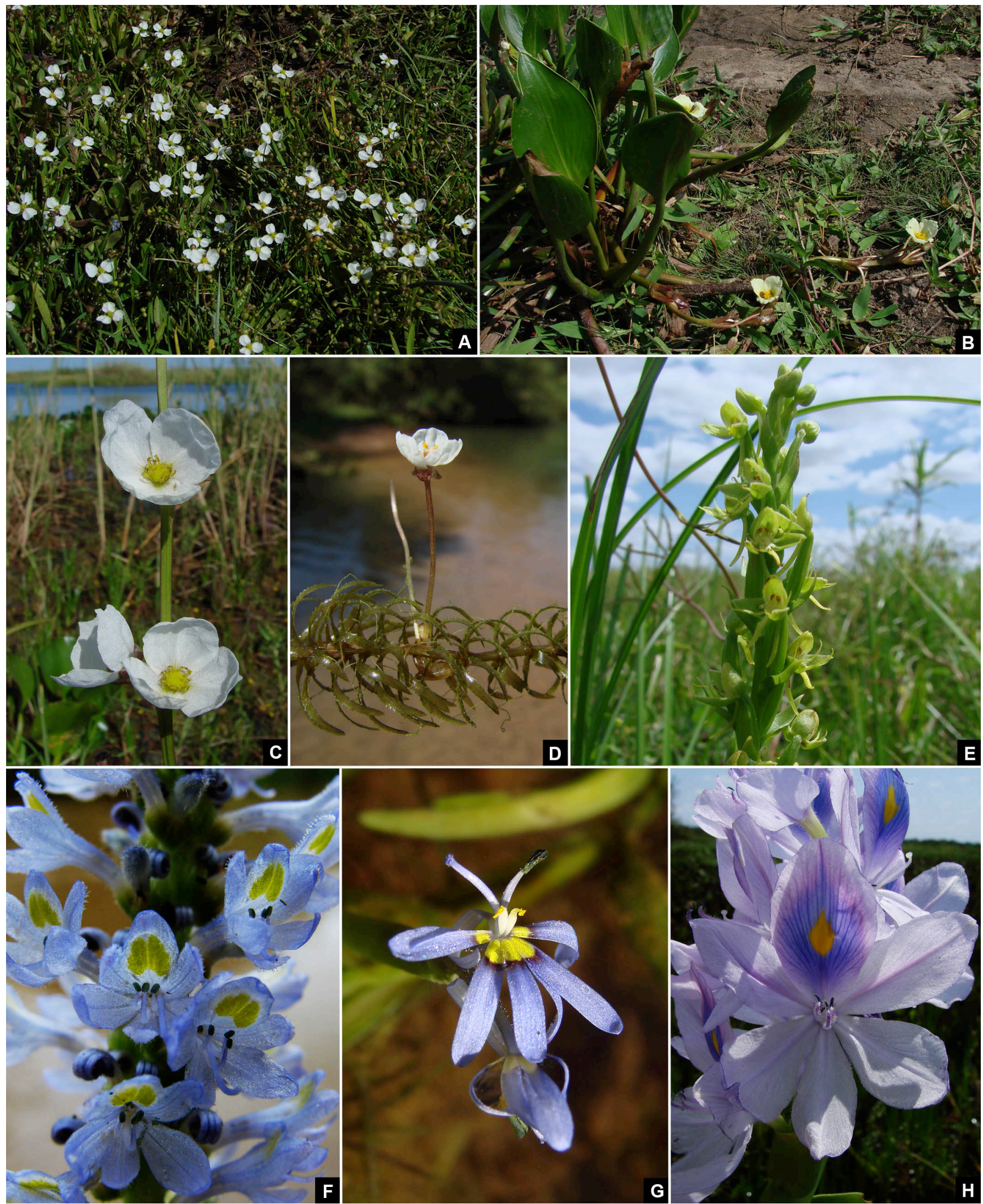

FigURE 4. Aquatic macrophyte species photographed at the Pandeiros River Wildlife Sanctuary: Monocotyledons. A. Helanthium bolivianum (Alismataceae), B. Sagittaria rhombifolia (Alismataceae), C. Echinodorus paniculatus (Alismataceae), D. Egeria najas (Hydrocharitaceae), E. Habenaria repens (Orchidaceae), F. Pontederia cordata (Pontederiaceae), G. Hydrothrix gardneri (Pontederiaceae), H. Eichhornia crassipes (Pontederiaceae). 

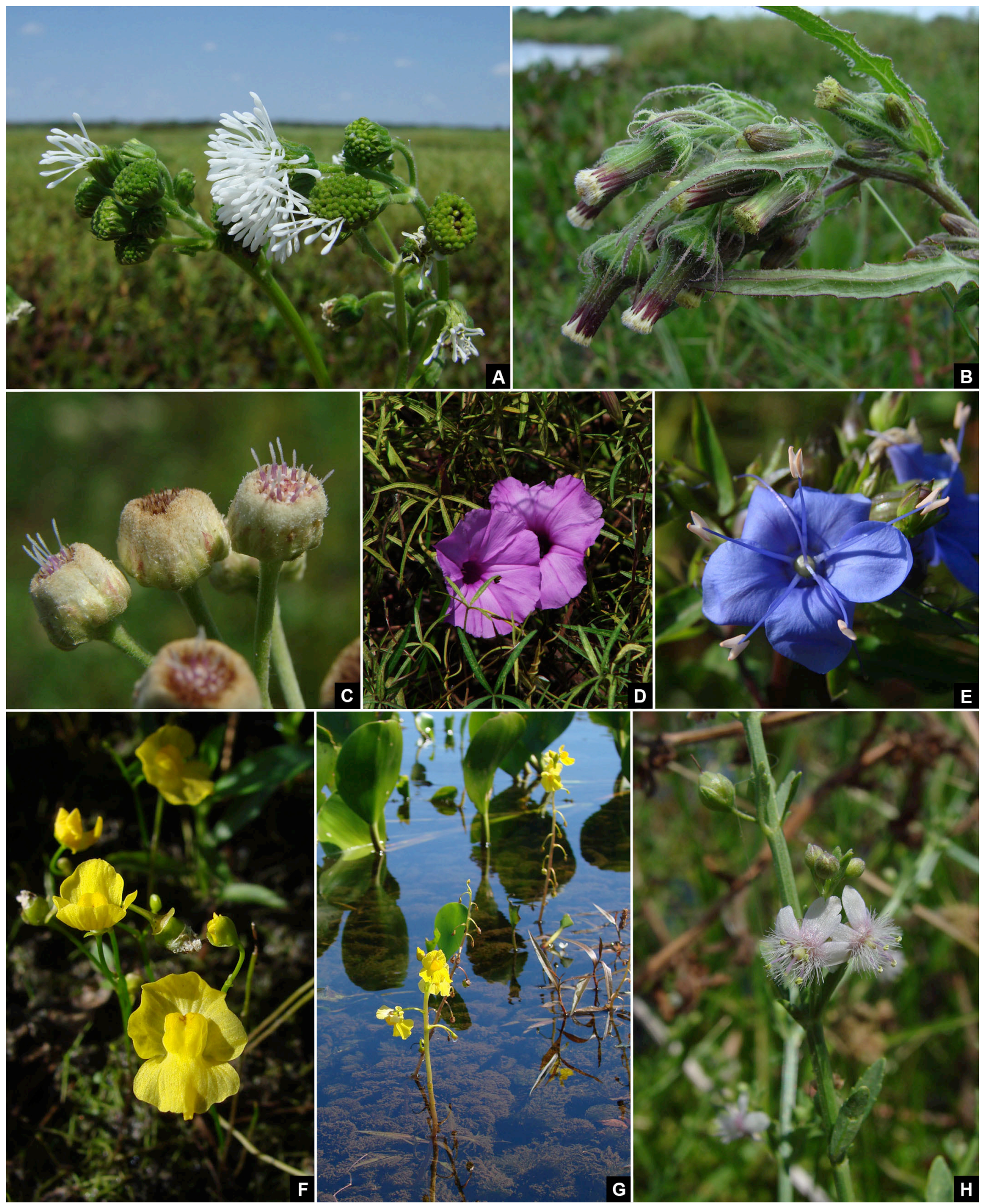

FiguRE 5. Aquatic macrophyte species photographed at the Pandeiros River Wildlife Sanctuary: Eudotyledons. A. Gymnocoronis spilanthoides (Asteraceae), B. Erechtites hieraciifolius (Asteraceae), C. Pluchea sagittalis (Asteraceae), D. Ipomoea sp. (Convolvulaceae), E. Hydrolea spinosa (Hydroleaceae), F. Utricularia gibba (Lentibulariaceae), G. Utricularia foliosa (Lentibulariaceae), H. Scoparia dulcis (Plantaginaceae). 

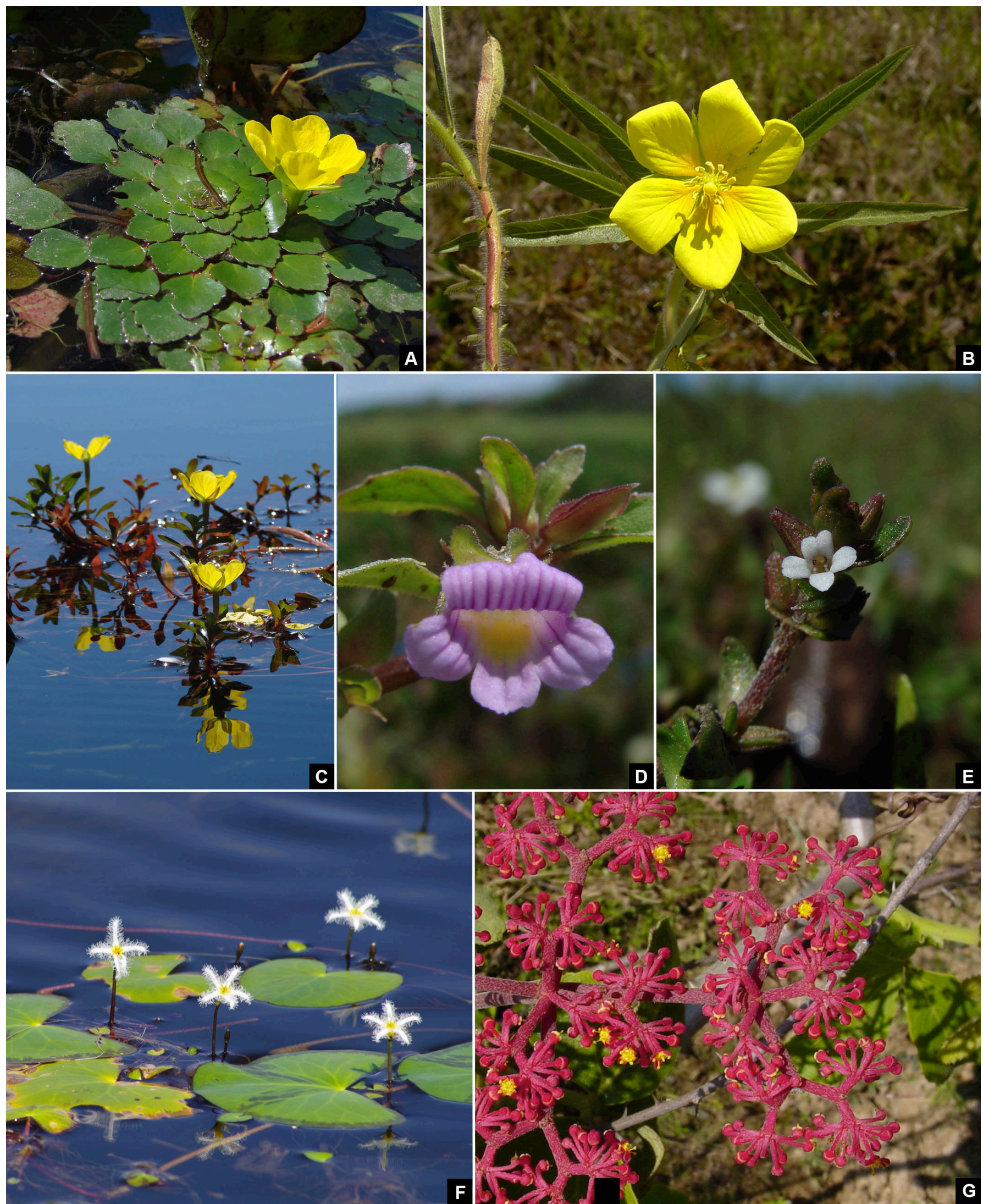

FIGURE 6. Aquatic macrophyte species photographed at the Pandeiros River Wildlife Sanctuary: Eudotyledons. A. Ludwigia sedoides (Onograceae), B. Ludwigia leptocarpa (Onograceae), C. Ludwigia inclinata (Onograceae), D. Cuphea racemosa (Lythraceae), E. Bacopa monnierioides (Plantaginaceae), F. Nymphoides indica (Menyanthaceae), G. Cissus spinosa (Vitaceae). 
TABLE 2. Aquatic macrophyte species of the Pandeiros River Wildlife Sanctuary in Minas Gerais, Brazil (* charophytes; ** liverworts; *** ferns). Life form: A- amphibian, Em- emerging, FF- fixed floating, FL- free floating, Rf- rafted plant, SF- fixed submerged and SL- free submerged. Voucher: (collector numbers) L- J.A. Lombardi, P- M.O.D. Pivari, S- A. Salino. Aquatic environments: PAN- Pandeiros riverbed; FLP- floodplains; OXL- oxbow lakes; SWAswamp.

\begin{tabular}{|c|c|c|c|c|c|c|}
\hline TAXON & LIFE FORM & VOUCHER & PAN & FLP & OXL & SWP \\
\hline \multicolumn{7}{|l|}{ Acanthaceae } \\
\hline Hygrophila cf. costata Nees & Em & P 1030 & & $\mathrm{x}$ & & \\
\hline Ruellia geminiflora Kunth & A & L 4810 & & & $\mathrm{x}$ & \\
\hline \multicolumn{7}{|l|}{ Alismataceae } \\
\hline Echinodorus paniculatus Micheli & A & P 558 & & $\mathrm{x}$ & $\mathrm{x}$ & \\
\hline Echinodorus subalatus (Mart. ex Schult.f.) Griseb. & A & P 1015 & & $\mathrm{x}$ & & \\
\hline Echinodorus grisebachii Small & A & P 1027 & & $\mathrm{x}$ & & \\
\hline Helanthium bolivianum (Rusby) Lehtonen and Myllys & Em & P 1025 & & $\mathrm{x}$ & & \\
\hline Sagittaria rhombifolia Cham. & A & P 557 & & & $\mathrm{x}$ & \\
\hline \multicolumn{7}{|l|}{ Amaranthaceae } \\
\hline Pfaffia tuberosa (Spreng.) Hicken & A & P 604 & & $\mathrm{x}$ & & \\
\hline \multicolumn{7}{|l|}{ Araceae } \\
\hline Pistia stratiotes L. & FL & L 4801 & & & $\mathrm{x}$ & \\
\hline \multicolumn{7}{|l|}{ Araliaceae } \\
\hline Hydrocotyle bonariensis Lam. & A & P 596 & & $\mathrm{x}$ & & \\
\hline \multicolumn{7}{|l|}{ Asteraceae } \\
\hline Baccharis genistelloides (Lam.) Pers. & A & P 1039 & & $\mathrm{x}$ & & \\
\hline Conocliniopsis prasiifolia (DC.) R.M.King and H.Rob. & Rf & P 597 & & & $\mathrm{x}$ & \\
\hline Eclipta prostrata (L.) L. & A & P 575 & & $\mathrm{x}$ & $\mathrm{x}$ & \\
\hline Erechtites hieraciifolius (L.) Raf. ex DC. & Rf & P 568 & & & $\mathrm{x}$ & \\
\hline Gymnocoronis spilanthoides (D.Don ex Hook. and Arn.) DC. & Rf & P 583 & & & $\mathrm{x}$ & \\
\hline Pluchea sagittalis Less. & Rf & P 593 & & & $\mathrm{x}$ & \\
\hline Vernonanthura phosphorica (Vell.) H.Rob. & $\mathrm{Rf}$ & P 609 & & & $\mathrm{x}$ & \\
\hline \multicolumn{7}{|l|}{ Cabombaceae } \\
\hline Cabomba furcata Schult. and Schult.f. & SF & P 1019 & & & $\mathrm{x}$ & \\
\hline \multicolumn{7}{|l|}{ Ceratophyllaceae } \\
\hline Ceratophyllum demersum L. & SL & P 578 & & & & $\mathrm{x}$ \\
\hline \multicolumn{7}{|l|}{ Characeae $^{*}$} \\
\hline Chara sp. 1 & $\mathrm{SF}$ & P 1024 & $\mathrm{x}$ & & & \\
\hline Chara sp. 2 & $\mathrm{SF}$ & P 1021 & $\mathrm{x}$ & & & \\
\hline \multicolumn{7}{|l|}{ Commelinaceae } \\
\hline Commelina cf. schomburgkiana Klotzsch & $\mathrm{Rf}$ & P 579 & & & $\mathrm{x}$ & \\
\hline Commelina sp. & A & P 594 & & $\mathrm{x}$ & & \\
\hline \multicolumn{7}{|l|}{ Convolvulaceae } \\
\hline Aniseia martinicensis (Jacq.) Choisy & Rf & P 586 & & & $\mathrm{x}$ & \\
\hline Ipomoea sp. & Rf & P 553 & & & $\mathrm{x}$ & \\
\hline \multicolumn{7}{|l|}{ Cyperaceae } \\
\hline Cyperus giganteus Vahl & Em & P 1053 & $\mathrm{x}$ & & & \\
\hline Cyperus haspan L. & A & P 562 & & $\mathrm{x}$ & $\mathrm{x}$ & \\
\hline Cyperus odoratus L. & A & P 1034 & & $\mathrm{x}$ & $\mathrm{x}$ & \\
\hline Cyperus surinamensis Rottb. & Rf & P 556 & & & $\mathrm{x}$ & \\
\hline Cyperus sp. & A & P 1048 & & $\mathrm{x}$ & & \\
\hline Eleocharis geniculata (L.) Roem. and Schult. & Em & P 1045 & & $\mathrm{x}$ & $\mathrm{x}$ & \\
\hline Eleocharis plicarhachis (Griseb.) Svenson & Rf & P 610 & & & $\mathrm{x}$ & \\
\hline Fuirena umbellata Rottb. & A & P 1031 & & $\mathrm{x}$ & & \\
\hline Kyllinga pumila Michx. & A & P 1026 & & $\mathrm{x}$ & & \\
\hline Oxycaryum cubense (Poepp. and Kunth) Palla & $\mathrm{Rf}$ & P 567 & & & $\mathrm{x}$ & \\
\hline \multicolumn{7}{|l|}{ Fabaceae } \\
\hline Aeschynomene sensitiva Sw. & Rf & P 572 & & & $\mathrm{x}$ & \\
\hline Dioclea virgata (Rich.) Amshoff & A & P 1057 & & $\mathrm{x}$ & & \\
\hline Erythrina speciosa Andrews & Em & P 1037 & $\mathrm{x}$ & & & \\
\hline Senna splendida (Vogel) H.S.Irwin and Barneby & Em & P 1042 & $\mathrm{x}$ & & & \\
\hline Vigna cf. lasiocarpa (Benth.) Verdc. & A & P 595 & & $\mathrm{x}$ & & \\
\hline Vigna sp. & A & P 617 & & & $\mathrm{x}$ & \\
\hline \multicolumn{7}{|l|}{ Hydrocharitaceae } \\
\hline Egeria najas Planch. & SF & P 1018 & & & & $\mathrm{x}$ \\
\hline
\end{tabular}


TABle 2. Continued.

\begin{tabular}{|c|c|c|c|c|c|c|}
\hline TAXON & LIFE FORM & VOUCHER & PAN & FLP & OXL & SWP \\
\hline \multicolumn{7}{|l|}{ Hydroleaceae } \\
\hline Hydrolea spinosa L. & A & P 559 & & & $\mathrm{x}$ & \\
\hline \multicolumn{7}{|l|}{ Lamiaceae } \\
\hline Hyptis pectinata (L.) Poit. & $\mathrm{Rf}$ & P 592 & & & $\mathrm{x}$ & \\
\hline Indeterminate & A & P 1051 & & $\mathrm{x}$ & & \\
\hline \multicolumn{7}{|l|}{ Lentibulariaceae } \\
\hline Utricularia gibba L. & Rf & P 566 & & & $\mathrm{x}$ & \\
\hline \multicolumn{7}{|l|}{ Lythraceae } \\
\hline Cuphea melvilla Lindl. & Rf & P 1032 & & & $\mathrm{x}$ & \\
\hline Cuphea racemosa (L.f.) Spreng. & $\mathrm{Rf}$ & P 582 & & & $\mathrm{x}$ & \\
\hline \multicolumn{7}{|l|}{ Melastomataceae } \\
\hline Rhynchanthera sp. & $\mathrm{Rf}$ & P 570 & & & $\mathrm{x}$ & \\
\hline Nymphoides indica (L.) Kuntze & A & L 4800 & & $\mathrm{x}$ & & \\
\hline \multicolumn{7}{|l|}{ Nymphaeaceae } \\
\hline Nymphaea lingulata Wiersema & $\mathrm{FF}$ & L 4812 & & & $\mathrm{x}$ & \\
\hline Nymphaea rudgeana G.Mey. & FF & P 1017 & & & & $\mathrm{x}$ \\
\hline \multicolumn{7}{|l|}{ Ochnaceae } \\
\hline Sauvagesia erecta L. & A & P 1033 & & $\mathrm{x}$ & & \\
\hline \multicolumn{7}{|l|}{ Onagraceae } \\
\hline Ludwigia inclinata (L.f.) M.Gómez & $\mathrm{SF}$ & P 1010 & & & & $\mathrm{x}$ \\
\hline Ludwigia leptocarpa (Nutt.) H.Hara & A & P 554 & & $\mathrm{x}$ & $\mathrm{x}$ & \\
\hline Ludwigia octovalvis (Jacq.) P.H.Raven & A & P 607 & & $\mathrm{x}$ & & \\
\hline Ludwigia sedoides (Humb. and Bonpl.) H.Hara & FF & Only photographed & & & $\mathrm{x}$ & \\
\hline Ludwigia cf. tomentosa (Cambess.) H. Hara & Rf & P 587 & & & $\mathrm{x}$ & \\
\hline \multicolumn{7}{|l|}{ Orchidaceae } \\
\hline \multicolumn{7}{|l|}{ Orobanchaceae } \\
\hline Melasma melampyroides (Rich.) Pennell ex Britton and P.Wilson & Rf & P 600 & & & $\mathrm{x}$ & \\
\hline \multicolumn{7}{|l|}{ Plantaginaceae } \\
\hline Bacopa monnierioides (Cham.) B.L.Rob. & Em & P 1029 & & & $\mathrm{x}$ & \\
\hline Bacopa salzmannii (Benth.) Edwall & A & L 4803 & & $\mathrm{x}$ & & \\
\hline Bacopa serpylloides (Cham. and Schltdl.) Angely & A & P 608 & & $\mathrm{x}$ & & \\
\hline Bacopa stricta (Schrad.) Wettst. ex Edwall & A & P 1050 & & $\mathrm{x}$ & & \\
\hline Bacopa sp. & $\mathrm{Rf}$ & P 564 & & & $\mathrm{x}$ & \\
\hline Scoparia dulcis L. & Rf & P 581 & & & $\mathrm{x}$ & \\
\hline \multicolumn{7}{|l|}{ Poaceae } \\
\hline Arundinella hispida (Willd.) Kuntze & A & P 1046 & & $\mathrm{x}$ & & \\
\hline Axonopus fissifolius (Raddi) Kuhlm. & A & P 1047 & & $\mathrm{x}$ & & \\
\hline Hymenachne pernambucensis (Spreng.) Zuloaga & A & P 1041 & & $\mathrm{x}$ & & \\
\hline Imperata tenuis Hack. & A & L 4808 & & $\mathrm{x}$ & & \\
\hline Leersia hexandra Sw. & $\mathrm{Em}$ & P 1013 & & & & $\mathrm{x}$ \\
\hline Panicum mertensii Roth & A & P 1040 & & $\mathrm{x}$ & & \\
\hline Paspalum repens P.J.Bergius & $\mathrm{FF}$ & L 4799 & & & $\mathrm{x}$ & \\
\hline Steinchisma laxa (Sw.) Zuloaga & A & P 1044 & & $\mathrm{x}$ & & \\
\hline Urochloa sp. & Rf & P 611 & & & $\mathrm{x}$ & \\
\hline \multicolumn{7}{|l|}{ Polygonaceae } \\
\hline Polygonum acuminatum Kunth & A & P 603 & & & & $\mathrm{x}$ \\
\hline Polygonum meisnerianum Cham. and Schltdl. & A & P 560 & & & $\mathrm{x}$ & \\
\hline Polygonum persicaria L. & Em & P 1012 & & $\mathrm{x}$ & & \\
\hline \multicolumn{7}{|l|}{ Pontederiaceae } \\
\hline Eichhornia azurea (Sw.) Kunth & FF & P 576 & $\mathrm{x}$ & & $\mathrm{x}$ & $\mathrm{x}$ \\
\hline Eichhornia crassipes (Mart.) Solms & FL & P 577 & & & & $\mathrm{x}$ \\
\hline Heteranthera seubertiana Solms & Em & $\mathrm{L} 4813$ & & $\mathrm{x}$ & & \\
\hline
\end{tabular}


TABLE 2. CONTINUED.

\begin{tabular}{|c|c|c|c|c|c|c|}
\hline TAXON & LIFE FORM & VOUCHER & PAN & FLP & OXL & SWP \\
\hline \multicolumn{7}{|l|}{ Potamogetonaceae } \\
\hline Potamogeton polygonus Cham. & SF & P 1020 & & & & $\mathrm{x}$ \\
\hline \multicolumn{7}{|l|}{ Pteridaceae $^{* * *}$} \\
\hline Ceratopteris pteridoides (Hook.) Hieron. & Em & P 588 & & & $\mathrm{x}$ & $\mathrm{x}$ \\
\hline Pityrogramma calomelanos (L.) Link & Rf & P 591 & & & $\mathrm{x}$ & \\
\hline Pityrogramma trifoliata (L.) R.M.Tryon & A & P 1056 & & $\mathrm{x}$ & & \\
\hline \multicolumn{7}{|l|}{ Ricciaceae $^{* *}$} \\
\hline Ricciocarpus natans (L.) Corda & FL & P 589 & & & $\mathrm{x}$ & $\mathrm{x}$ \\
\hline \multicolumn{7}{|l|}{ Rubiaceae } \\
\hline Borreria capitata (Ruiz and Pav.) DC. & A & P 1049 & & $\mathrm{x}$ & & \\
\hline Borreria scabiosoides Cham. and Schltdl. & $\mathrm{Rf}$ & P 585 & & & $\mathrm{x}$ & \\
\hline Borreria spinosa (L.) Cham. and Schltdl. & A & P 555 & & $\mathrm{x}$ & & \\
\hline Diodella cf. sarmentosa (Sw.) Bacigalupo and Cabral ex Borhidi & $\mathrm{Rf}$ & P 563 & & & $\mathrm{x}$ & \\
\hline Galianthe brasiliensis (Spreng.) E.L.Cabral and Bacigalupo & A & P 606 & & $\mathrm{x}$ & & \\
\hline \multicolumn{7}{|l|}{ Salviniaceae $* * *$} \\
\hline Azolla filiculoides Lam. & FL & S 8013 & & & & $\mathrm{x}$ \\
\hline Salvinia auriculata Aubl. & FL & P 590 & & & $\mathrm{x}$ & $\mathrm{x}$ \\
\hline Salvinia oblongifolia Mart. & FL & P 584 & & & $\mathrm{x}$ & $\mathrm{x}$ \\
\hline \multicolumn{7}{|l|}{ Thelypteridaceae $* * *$} \\
\hline Thelypteris interrupta (Willd.) Iwats. & A & P 1058 & & $\mathrm{x}$ & & \\
\hline \multicolumn{7}{|l|}{ Verbenaceae } \\
\hline Lantana cf. hypoleuca Briq. & A & P 601 & & $\mathrm{x}$ & & \\
\hline \multicolumn{7}{|l|}{ Vitaceae } \\
\hline Cissus spinosa Cambess. & A & P 605 & $\mathrm{x}$ & & & \\
\hline
\end{tabular}

ACKNOWLeDgments: We are thankful for Chico de Souza and Pedro de Juca for logistical support in the fieldwork expeditions. Aristônio Magalhães Teles, Julio Antonio Lombardi, Alexandre Salino, Cecília Gontijo Leal, Leonardo Esteves Lopes and Gabriel Pescador were helpful during the collection of botanical samples. Alex Popovkin for help with the english version and Alexandre Salino and Suzana Neves Moreira for manuscript reviews. We also thank IEF-MG (Minas Gerais State Forestry Institute) for providing the collection permit for the Pandeiros River Wildlife Sanctuary and the curator of BHCB Herbarium for making the specimens available for study.

\section{Literature Cited}

APG III - Angiosperm Phylogeny Group. 2009. An update of the Angiosperm Phylogeny Group classification for the orders and families of flowering plants. Botanical Journal of the Linnean Society 161: 105-121.

Barbosa, I.S. and P. Maillard. 2010. Mapping a wetland complex in the Brazilian savannah using an Ikonos image: assessing the potential of a new region-based classifier. Canadian Journal of Remote Sensing 36(2): 231-242.

Bove, C.P., A.S.B. Gil, C.B. Moreira, C.B. and R.F.B. Anjos. 2003. Hidrófitas fanerogâmicas de ecossistemas aquáticos temporários da planície costeira do estado do Rio de Janeiro, Brasil. Acta Botanica Brasilica 17(1): 119-135.

Brummitt, R.K. and C.E. Powell. 1992. Authors of plant names. Kew: Royal Botanic Garden. 732 p.

Ceska, A. 1986. More on the techniques for collecting aquatic and marsh plants. Annals of the Missouri Botanical Garden 73: 825-827.

Conservação Internacional. 1999. Ações prioritárias para a biodiversidade do cerrado e pantanal. Brasília. $26 \mathrm{p}$.

Drummond, G.M., C.S. Martins, A.B.M. Machado, F.A. Sebaio and Y. Antonini. 2005. Biodiversidade em Minas Gerais: um atlas para sua conservação. Belo Horizonte: Fundação Biodiversitas. 222 p.

Duzer, C.V. 2004. Floating islands - A global bibliography: with an edition and translation of G.C. Munz's Exercitatio academica de insulis natantibus (1711). California: Cantor Press. 404 p.

Esteves, F.A. 1988. Fundamentos de Limnologia. Rio de Janeiro: Interciência. 602 p.

Haynes, R.R. 1984. Techniques for collecting aquatic and marsh plants. Annals of the Missouri Botanical Garden 71: 229-231.

Irgang, B.E., G. Pedralli and J.I. Waechter. 1984. Macrófitos aquáticos da Estação Ecológica do Taim, Rio Grande do sul, Brasil. Roessleria 6: 395-404.

Kohler, H.C. 2003. Aspectos geoecológicos da bacia hidrográfica do São
Francisco (primeira aproximação na escala 1:1000000); p. 25-35 In H.P. Godinho and A.L. Godinho (ed.). Águas, peixes e pescadores do São Francisco das Minas Gerais. Belo Horizonte: Editora PUC Minas.

Köppen, W. 1931. Climatologia. Buenos Aires: Fondo de Cultura Económica. 390 p.

Kramer K.U. and Green P.S. 1990. The families and genera of vascular plants. Pteridophytes and Gymnosperms. New York: Springer Verlag. $404 \mathrm{p}$.

Lombardi, J.A., A. Salino and L.G. Temponi. 2005. Diversidade florística de plantas vasculares no município de Januária, Minas Gerais, Brasil. Belo Horizonte. Lundiana: 6(1): 3-20.

Lorenzi, H. 2000. Plantas daninhas do Brasil: terrestres, aquáticas, parasitas e tóxicas. Nova Odessa: Instituto Plantarum. 640 p.

Nunes, Y.R.F., I.F.P. Azevedo, W.V. Neves, M.D.M. Veloso, R.A.S. Souza and G.W. Fernandes. 2009. Pandeiros: o Pantanal Mineiro. MG Biota 2: 4-17.

Pivari, M.O.D., V.B. Oliveira, F.M. Costa, R.M. Ferreira and A. Salino. 2011. Macrófitas aquáticas do sistema lacustre do Vale do Rio Doce, Minas Gerais, Brasil. Rodriguésia 62(4): 759-770.

Pivari, M.O.D., V.J. Pott and A. Pott. 2008. Macrófitas aquáticas de ilhas flutuantes (baceiros) nas sub-regiões do Abobral e Miranda, Pantanal, MS, Brasil. Acta Botanica Brasilica 22(2): 559-567.

Pott, V.J. and A. Pott. 2000. Plantas aquáticas do Pantanal. Corumbá: Embrapa. 353 p.

Ratter, J.A., G.P. Askew, R.F. Montgomery and D.R. Gifford. 1978. Observations on forests of some mesotrophic soils in Central Brazil. Revista Brasileira de Botânica 1: 47-58.

Raven, H.P., R.F. Evert and S.E. Eichhorn. 1996. Biologia Vegetal. Rio de Janeiro: Guanabara Koogan. 906 p.

Rebouças, A.C., B. Braga and J.G. Tundisi. 1999. Águas doces no Brasil capital ecológico, uso e conservação. São Paulo: Escrituras Editora. $717 \mathrm{p}$.

Sato, Y. and H.P. Godinho. 2003. Migratory fishes of the São Francisco River; p. 199-232 In J. Carolsfeld, B. Harvey, C. Ross and E.A. Baer (ed.). Migratory fishes of South America: biology, fisheries and conservation status. Victoria: World Fisheries Trust/IDRC/World Bank.

Thiers, B. 2012. Index Herbariorum: A global directory of public herbaria and associated staff. Eletronic Database accessible at http:// sweetgum.nybg.org/ih/. Captured on 11 October 2012.

RECEIVED: November 2012

ACCEPTED: February 2013

Published ONLINE: April 2013

EDITORIAL RESPONSIBILITY: Frederico Augusto Guilherme 